\title{
Rhythmic context modulates foreperiod effects
}

\author{
ROBERT J. ELLIS \\ Ohio State University, Columbus, Ohio \\ and Beth Israel Deaconess Medical Center and Harvard Medical School, Boston, Massachusetts \\ AND \\ MARI RIESS JONES \\ Ohio State University, Columbus, Ohio \\ and University of California, Santa Barbara, California
}

\begin{abstract}
Two experiments examined hypotheses about the roles of probabilistic uncertainty and rhythmic context on attentional preparation as reflected by choice response times (RTs) to the final tone of auditory sequences. Nonisochronous sequences with tone timings either arranged metrically or scrambled were linked with one of three different sequence-final time intervals, or foreperiods (FPs), which varied randomly from trial to trial. Two primary results emerged. First, RTs were faster to target tones ending metrical rhythms than to targets ending scrambled rhythms. Second, metrical contexts elicited RTs that increased with FP duration, whereas scrambled contexts elicited RTs that often decreased with FP duration, despite equivalent variability of time intervals in metrical and scrambled contexts. The results suggest that time relations implied by metrical rhythms systematically modulate preparatory responses to sequence-final FPs.
\end{abstract}

Preparing for a future event involves expectations about both the what and the when of that event. In the present study, we considered two very different perspectives on the role of temporal contexts in eliciting such preparatory expectations. A classic view derives from the foreperiod (FP) literature, in which responses to single time intervals have been studied. Different FP paradigms permit examination of the relationship between probabilistic uncertainty and reaction time (RT). A common assumption is that low uncertainty about the what and when of a forthcoming time interval, conferred probabilistically by prior trial context, facilitates preparatory activity. A different perspective derives from research on sequence perception. It is concentrated on relational (rather than probabilistic) properties of time intervals within sequences that facilitate preparation of forthcoming time intervals.

In the present study, we outline contrasting hypotheses about the role of temporal context that emerge from these two perspectives. To evaluate these hypotheses, we modified a standard FP paradigm by incorporating each of three FPs $(250,500$, and $1,000 \mathrm{msec})$ as the final time interval of rhythmical auditory patterns that varied in degree of temporal coherence.

\section{Uncertainties in FP Contexts}

Classically, temporal preparation for a forthcoming target event has been studied by varying the duration of a single time interval (i.e., an FP) that occurs between a warning signal and a target on each of a series of trials. ${ }^{1}$ Faster RTs to the target presumably indicate better temporal preparation to a particular FP. Conventionally, an RT to a given FP depends on two features: FP duration and the intertrial context in which the FP occurs (e.g., Niemi \& Näätänen, 1981).

Intertrial (or global) context differentiates the two major paradigms used to study temporal preparation: In a variable-FP design, FPs vary from trial to trial within a block; in a constant-FP design, a single FP is used within a trial block. In the variable-FP paradigm, the probability distribution of FPs over trials is assumed to determine the uncertainty of an FP on an individual trial. In the simplest case, a uniform probability distribution of different FPs typically yields RTs that get shorter as an FP lengthens: a decreasing RT-FP function. The most parsimonious explanation for this function is that uncertainty decreases as time elapses from a warning signal, and this decreased uncertainty results in shorter RTs to longer FPs (e.g., Klemmer, 1956; Niemi \& Näätänen, 1981; Woodrow, 1914). A flat RT-FP function is also consistent with a uniform FP distribution, but suggests that the subjects acquired little knowledge of FPs from the global context - that is, that uncertainty was uniformly high for all FPs.

The constant-FP paradigm yields an RT-FP function that is characteristically different from that of variable-FP profiles, with RTs lengthening as FP lengthens. Such ascending RT-FP profiles are attributed to subjects' reduced abilities to estimate longer (albeit predictable) time intervals (Correa, Lupiáñez, \& Tudela, 2005; Klemmer, 1956; Steinborn, Rolke, Bratzke, \& Ulrich, 2008). 
A common explanation for these opposing RT-FP profiles appeals to uncertainty: Higher uncertainty leads to poorer preparation and slower responding. To explain the respective role of uncertainty in variable- and constant-FP paradigms, Klemmer (1956) distinguished two types of uncertainty - namely, uncertainty about what and when. Uncertainty about what refers to which of several FPs might occur on a given trial, whereas uncertainty about when refers to uncertainty about the ending time of a predictable FP. Klemmer maintained that the variable-FP paradigm induces uncertainty about both the what of an FP and when the FP will end, whereas the constant-FP paradigm results in uncertainty only about when. Moreover, in the latter, high temporal uncertainty reflects increasing subjective difficulty in time estimation with longer FPs. Thus, Weber's law can describe increasing RTs to longer FPs within an FP set.

Importantly, Klemmer's (1956) explanation means that only uncertainty about what is affected by probabilistic uncertainty. Probabilistic uncertainty correctly describes RT-FP profiles of the variable-FP paradigm (with FPs over $200 \mathrm{msec}$ ). For instance, reductions in probabilistic uncertainty as an FPs time elapses nicely predicts the finding that perceivers respond most slowly to the shortest FP and most quickly to the longest one (Drazin, 1961; Niemi \& Näätänen, 1981). However, it is not uncommon in the variable-FP paradigm to also find that perceivers learn the length of the longest FP and attempt to estimate its onset. Because time estimation covaries with elapsed time (per Weber's law; for a discussion, see Grondin, 2001), RTs to longer FPs may become longer, leading to a U-shaped RT-FP profile in the variable-FP paradigm (Klemmer, 1956; Niemi \& Näätänen, 1981). This quadratic trend reinforces Klemmer's claim that uncertainty about what is absent for the longest FP of set.

The preceding FP research documents a common finding: an interaction of intertrial context (variable FP, constant FP) with FP (short, medium, long) that is manifest in opposing linear trends in the two RT-FP profiles (Klemmer, 1956; Müller-Gethmann, Ulrich, \& Rinkenauer, 2003; Niemi \& Näätänen, 1981; Woodrow, 1914). Less research has been focused on the effects of local, or intratrial, context: stimuli that immediately precede a warning signal (i.e., a precue) or that fill an FP interval. Both kinds of local context appear to influence RTs. However, most of this research suggests the ubiquity of a decreasing RT-FP profile, consistent with the variable-FP paradigm, over different local context manipulations. For instance, Bertelson (1967; Bertelson \& Tisseyre, 1968) used a precue design to examine choice RTs (to FPs less than $300 \mathrm{msec}$ ). $\mathrm{He}$ introduced uncertainty in the timing of a warning signal (either $1.5-5$ or $5 \mathrm{sec}$ across conditions) following a single precue. In both the constant-FP and the variable-FP paradigms, RTs decreased as FPs lengthened.

Others have examined intratrial context by filling FPs with isochronous or simple rhythms. Requin, Grandjon, Durup, and Reynard (1973; cf. Simon \& Slaviero, 1975) filled six FP intervals (from 4 to $24 \mathrm{sec}$ ) with isochronous clicks that varied in rate using the variable-FP paradigm. When click rate matched certain FPs, a classic decreasing RT-FP pro- file (using simple rather than choice RTs) emerged. Martin et al. (2005, Experiment 3; cf. Martin et al., 2006) reported that choice RTs to visual targets were shorter when those targets were part of simple rhythms, which they explained using an entrainment model (cf. Large \& Jones, 1999). Unlike the present study, however, Martin et al. (2005, Experiment 3) did not examine the role of final interonset time intervals (IOIs; i.e., the FP) in modulating RTs.

In summary, the foreperiod literature indicates that RTs to individual FPs are systematically influenced by global temporal context, defined by particular probability distributions of unrelated time intervals. Few systematic manipulations of other temporal aspects of these distributions have been examined. Other than Martin et al. (2005, Experiment 3), manipulations of rhythmic context have been confined to isochronous (Correa \& Nobre, 2008; Martin et al., 2005, Experiment 1; Requin et al., 1973) or isochronous versus irregular rhythms (Doherty, Rao, Mesulam, \& Nobre, 2005; Olson \& Chun, 2001), which differ markedly in their statistical, grouping, and metrical properties. Accordingly, we asked what happens if temporal contexts differ in rhythmic coherence but retain a common probability structure.

\section{Rhythmic Contexts}

To answer this question, we considered an alternative perspective on temporal preparation, one that is focused on relationships among time intervals. This orientation is common in the literature on rhythm perception. In listening to a sequence of tones, one tends to anticipate future tone onsets on the basis of preceding temporal regularities. Defined broadly, a rhythm is simply a serial arrangement of time intervals; within this frame, rhythms may vary greatly in temporal regularity/coherence. Our aim was to manipulate the serial arrangement of a given set of time intervals to create temporal contexts for FPs that vary in rhythmic coherence but that retain equivalent statistical properties.

To this end, it is necessary to identify pattern features responsible for coherent rhythmic relationships. The most recognized examples typically involve rhythmic regularity manifest as isochrony, where all IOIs are identical. Although isochrony is the most studied temporal regularity, it is not the one most commonly encountered in daily environments. Rather, nonisochronous rhythms are more prevalent, with some conveying significant temporal coherence despite temporal variability. Indeed, certain nonisochronous rhythms may be more effective in eliciting temporal anticipations than isochronous ones (Ellis \& Jones, 2009; Large, 2010; Lerdahl \& Jackendoff, 1983; London, 2004; Martin et al., 2005; Snyder \& Krumhansl, 2001; Temperley, 2001). Although it remains unclear exactly what aspects of a rhythm confer temporal coherence, various candidates have been suggested. For example, Gestalt approaches propose that homogeneous, recurrent groups of tones within a sequence offer salient regularities (e.g., Bregman, 1990; Garner, 1974; Handel, 1992); others suggest a role for first-order conditional variability of time intervals (Patel \& Daniele, 2003; Patel, Iversen, \& Rosenberg, 2006), general statistical properties (Drake \& Botte, 1993), or metrical relationships (Fraisse, 
1978; Jones, 1976, 2009; London, 2004; Povel \& Essens, 1985 ) in defining salient rhythmic properties.

The present investigation is focused on metrical relationships as a basis for rhythmic coherence in tone patterns for two reasons. First, one approach to meter perception links it to temporal expectancies and attentional preparation (i.e., anticipatory attending; Jones, 1976; Large \& Jones, 1999; Large \& Kolen, 1994). Second, metrical manipulations permit an assessment of rhythmic coherence due to implied time relationships in nonisochronous sequences, a topic that has received little attention.
Figure 1 presents two different categories of nonisochronous sequences that differ in rhythmic coherence: metrical (M) and scrambled (S). Both sets contain the same IOIs, where IOI refers to the time span between onsets of adjacent tones. All Figure 1 patterns contain the same basic set of IOIs $(250,500,750$, and $1,000 \mathrm{msec})$. However, the time intervals of $\mathrm{M}$ patterns are serially arranged such that accents (solid circles) occur regularly. The serial arrangement of IOIs in a given M pattern is haphazardly rearranged to create a yoked $\mathrm{S}$ sequence. Although these patterns are all nonisochronous, we maintain that $\mathrm{M}$ pat-

A

Metrical (M) Sequences

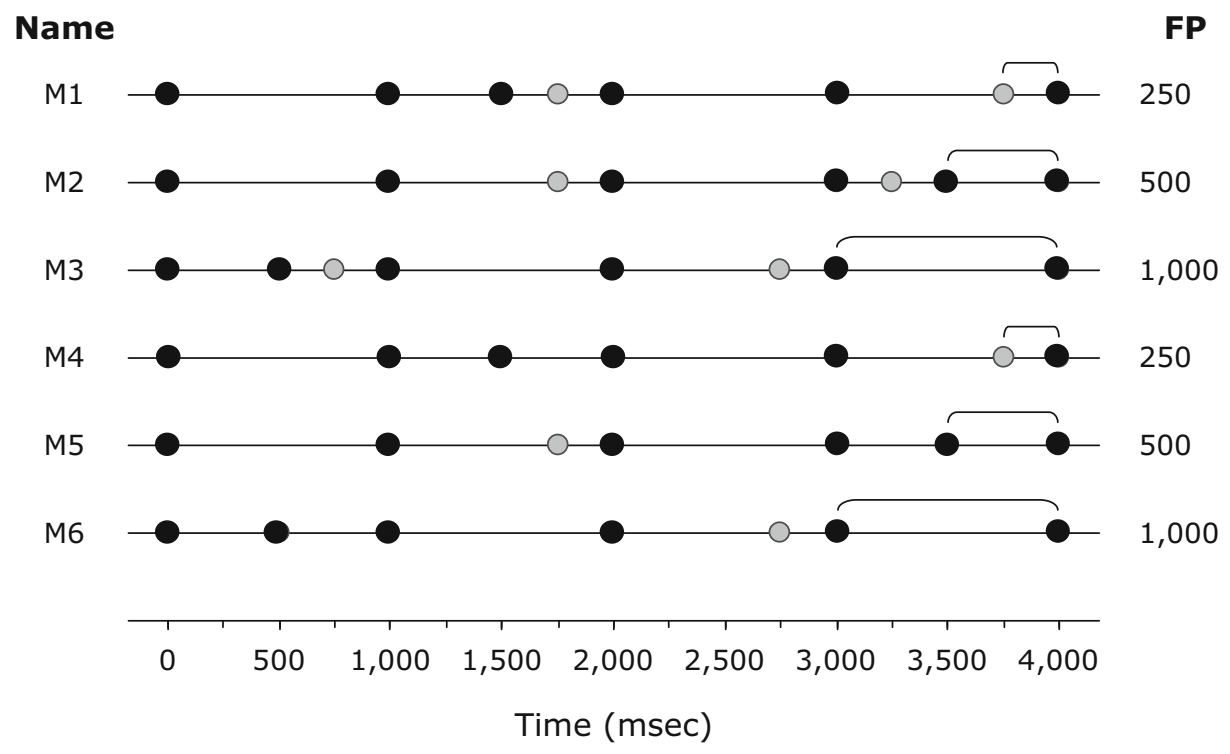

B Scrambled (S) Sequences

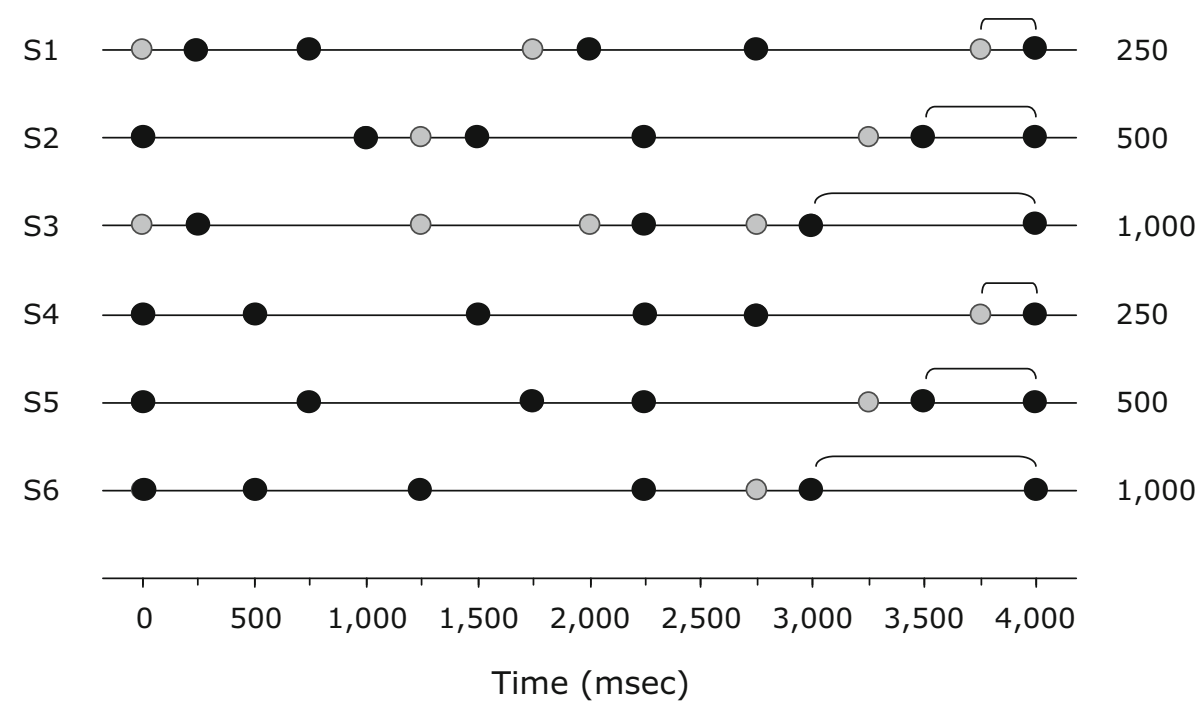

Figure 1. Arrangements of interonset intervals (IOIs) for metrical (M, panel A) and scrambled (S, panel B) sequences. IOIs were $250,500,750$, or 1,000 msec. Accented tones (see note 1) are shown as black solid circles and unaccented tones as gray circles. Sequences M1-M3 and S1-S3 have eight tones; the others have seven tones. The final IOI (foreperiod) is indicated by a bracket. 


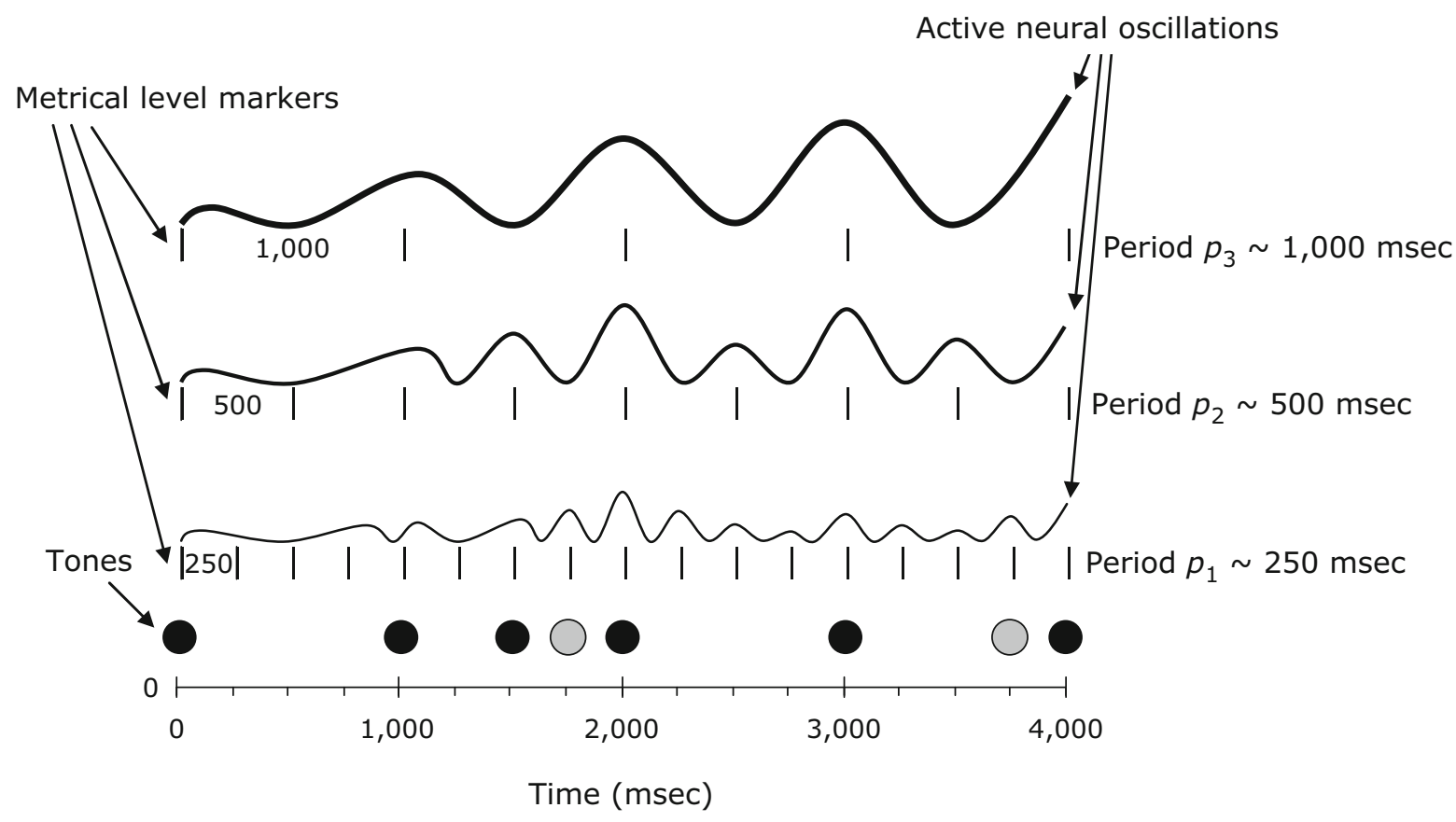

Figure 2. Multiple (nested) stimulus time levels for pattern M1 of Figure 1. Implied accents are consistent with a duple (4/4) meter and are shown as black vertical bars that separate recurrent time spans of 250, 500, and 1,000 msec. These reflect three stimulus time levels that function as stimulus-driving rhythms. Also shown, as wavy lines, are active neural oscillations, hypothesized to be internal beat periods. Each beat period instantiates an implied time period based on an entraining oscillator with a period, $p_{i}$. In entrainment theory, each oscillator adapts to match one of the stimulus-driving rhythms (250, 500, or $1,000 \mathrm{msec})$.

terns have greater rhythmic coherence than $\mathrm{S}$ sequences. Accents in both $\mathrm{M}$ and $\mathrm{S}$ patterns mark time spans between onsets of nonadjacent tones. Only in M patterns, however, do accents imply temporal regularity by marking an implied isochrony at each of several related time scales.

Figure 2 illustrates the implied time hierarchy present in all M patterns (using the exemplar pattern M1). Vertical lines indicate implied accent isochronies that correspond to each nested time scale $(250,500$, and 1,000 msec). By contrast, accents are haphazardly scrambled in S patterns to obscure nested time levels, reducing their rhythmic coherency. Three features of these two sequence categories are noteworthy: First, the same metrical relations are invariant over all $\mathrm{M}$ patterns. All of them conform to a common 4/4 Western duple meter, meaning that embedded time scales observe 2:1 ratios (i.e., 1,000/500/250 msec). Second, although pairs of $\mathrm{M}$ and $\mathrm{S}$ patterns differ in metrical constraints, they are equivalent probabilistically: They have identical IOI frequency distributions. Third, equally often, $\mathrm{M}$ and $\mathrm{S}$ patterns terminate with one of three sequence-final time intervals: 250,500 , or $1,000 \mathrm{msec}$. These final IOIs serve as FPs (highlighted by brackets in Figure 1), and each FP has the same probabilistic likelihood in $\mathrm{M}$ and $\mathrm{S}$ patterns.

The sequences of Figure 1 permit an adaptation of the variable-FP paradigm by placing FPs within a rhythmic context. Using a variable-FP choice RT paradigm, we addressed two questions. First, does a metrical (vs. a scrambled) rhythmic context more effectively prepare a listener to respond to final (target) tone? Second, do categorical differences between $\mathrm{M}$ and $\mathrm{S}$ rhythmic contexts differentially prepare listeners to respond to particular sequencefinal time interval (i.e., a given FP)?

Dynamic attending theory (DAT; Jones, 2009; Large \& Jones, 1999) offers an affirmative answer to the first question. The presence of metrical relations (M patterns) is proposed to prepare listeners better than the absence of these relations (S patterns) for the onset time of a final target, because metrical relationships promote attentional entrainment. In M patterns, different recurrent (nested) time spans simultaneously entrain corresponding neural oscillations that emerge early in a sequence as beat periods. This instantiates an early emergence of preparatory activities (anticipatory attending). Early emergence of internalized beat periods is predicted to result in fast responding to target tones of $\mathrm{M}$ patterns. Because $\mathrm{S}$ pattern structure does not imply nested time spans, no beat periods are activated and preparatory activity is weak, leading to relatively slow responses to targets. In summary, DAT predicts generally faster responding to $\mathrm{M}$ than to $\mathrm{S}$ patterns.

The second question concerns a possible interaction of rhythmic context with final FP, based on differential responding to specific FPs that end M patterns. A key principle is oscillator congruence: a matching of an oscillator period with a sequence IOI. In this regard, the earlier distinction between IOIs (time spans between adjacent tones) and time spans that are not IOIs (between nonadjacent tones; e.g., accents) is useful. In M patterns, each of three induced oscillations has a period of 250,500 , or $1,000 \mathrm{msec}$ and is maximally congruent with one final IOI (i.e., an FP 
of 250,500 , or $1,000 \mathrm{msec}$ ). For example, in the M1 pattern of Figure 2, the final IOI (FP) of $250 \mathrm{msec}$ is maximally congruent with the oscillation with a 250 -msec period; that is, it affords a 1:1 period match. The oscillations (and their variability) induced by $M$ patterns can influence a listener's RTs to specific FPs. Specifically, the variability of an induced oscillation is time scaled to its period (given entrainment assumptions), consistent with Weber's law. In turn, the variability of an oscillator that is congruent with a final FP determines a listener's RT to that FP. RTs thus increase with FP duration in M patterns. But because S patterns do not induce internal beat periods, RTs to the same FPs ending S patterns will not positively covary with FPs. In summary, in addition to predicting a main effect of rhythmic context, DAT also implies an interaction of rhythmic context with FP, wherein RTs positively covary with FP durations of M patterns but not with FP durations of S patterns. We elaborate on this idea in the General Discussion section.

In an attempt to integrate FP and rhythmic approaches, we incorporated $\mathrm{M}$ and $\mathrm{S}$ patterns as context rhythms into a variable-FP paradigm. Two different rhythm categories $(\mathrm{M}, \mathrm{S})$ were crossed with three final FPs $(250,500$, $1,000 \mathrm{msec}$ ). In Experiment 1, in an initial baseline condition, we used two-tone FP sequences in the first block and either M or S rhythms (with final FPs) in the second block. In Experiment 2, we pursued M and S context effects using a counterbalanced design.

Two hypotheses address the role of global context in these experiments. One is the probabilistic uncertainty hypothesis. It states that, as listeners learn a set of fixed FPs, uncertainty about what decreases with the elapsed time of an FP and RTs correspondingly decline. Relatedly, if learning about an FP set is hindered, uncertainties do not decline with FP duration; this results in long RTs and a flat RT-FP profile. Importantly, if the probability distributions (over trials) of time intervals for two different global contexts are identical, RTs and RT-FP profiles for these contexts should be identical. In our experiments, global contexts are provided by two precursor rhythm categories $(\mathrm{M}, \mathrm{S})$, which have statistically identical frequency distributions of time intervals. In a variable-FP paradigm, the probabilistic uncertainty hypothesis predicts a decreasing RT-FP profile for both M and S contexts with RTs negatively covarying with FPs, regardless of rhythm.

The DAT hypothesis holds that relational aspects of global contexts will differentiate responses to $M$ versus $\mathrm{S}$ rhythms despite equivalent probabilistic properties. Because of entrainment, metrical time relationships should lead to generally faster responses to $\mathrm{M}$ than to $\mathrm{S}$ patterns. In addition, congruence between sequence-final FP intervals $(250,500$, and $1,000 \mathrm{msec})$ and entrained beat periods ( 250 , 500 , and $1,000 \mathrm{msec}$ ) in $\mathrm{M}$ patterns should elicit specific temporal expectancies about when a final FP will end. This predicts a positive RT-FP profile for M patterns but not for S patterns.

\section{EXPERIMENT 1}

One goal of Experiment 1 was to confirm that our two-tone FP stimuli elicit a decreasing RT-FP profile.
In an initial trial block, three different FPs $(250,500$, or $1,000 \mathrm{msec}$ ) were presented randomly over trials as a baseline FP condition (FPonly). A second goal was to assess rhythmic context using these same two-tone stimulus pairs as the final tone pair of a precursor rhythm. In a second trial block, different groups of subjects received $\mathrm{M}$ and S precursor rhythms for the same three FPs, again utilizing a variable-FP paradigm.

According to the probabilistic uncertainty hypothesis, both blocks of Experiment 1 should produce a decreasing RT-FP function; regardless of the presence (or absence) of a rhythmic context, RTs should become shorter as FP lengthens. The DAT hypothesis addresses only the second trial block. DAT predicts a main effect of rhythmic context with shorter RTs to $\mathrm{M}$ versus $\mathrm{S}$ patterns and an interaction of rhythmic context with FPs: The RT-FP function should decrease for S patterns and increase for M patterns.

\section{Method}

Subjects. Thirty-two introductory psychology students from The Ohio State University served as subjects in return for course credit. All of them reported normal hearing and less than 5 years of formal musical training. They were randomly assigned to one of two conditions ( $\mathrm{M}$ or $\mathrm{S}$ patterns in Block 2), with 16 subjects in each.

Apparatus. A Dell Optiplex GX620 running E-Prime 1.1.4.1 (Psychology Software Tools, Sharpsburg, PA) controlled the experiment. The subjects listened to stimuli at a comfortable listening level through Sennheiser HD280 headphones and made responses on a standard USB computer keyboard.

Stimuli. The stimuli were sequences of sine tones created in Audacity 1.3.3 (http://audacity.sourceforge.net/). In the FPonly condition, a single induction tone $\left(\mathrm{E} 5, \mathrm{~F}_{\sharp} 5\right.$, or $\left.\mathrm{G}_{\sharp} 5\right)$ was followed by a yoked target at \pm 1 semitone, which occurred 250,500 , or $1,000 \mathrm{msec}$ after the onset of the induction tone. In the $\mathrm{M}$ and $\mathrm{S}$ conditions, monotonic precursor patterns of six tones (on the same three pitches as in the FPonly condition) were followed by yoked targets. Durations of induction and target tones were 25 and $40 \mathrm{msec}$, respectively, including a 5 -msec fade in/fade out. Eighteen sequences [3 (warning tones) $\times 2$ (yoked target tones) $\times 3($ FPs $)]$ occurred three times in a random order over trials.

Precursor patterns were permutations of the set of IOIs, corresponding to the seven-tone sequences in Figure 1 (M4-M6, S4-S6). In $\mathrm{M}$ patterns, IOIs were arranged to fit a strong $4 / 4$ meter. Tones with subjective accents ${ }^{2}$ (following Povel \& Okkerman, 1981; solid black circles in Figure 1) occurred either at the 1,000-msec period or at the 500-msec subdivision of it, creating sequences characterized as highly metrical by Povel and Essens (1985). Each S pattern was a rearrangement of the IOIs in a corresponding M pattern; few S patterns had metrically strong accents marking 1,000- or 500-msec periods. Consequently, the S patterns were nonmetrical (Povel \& Essens, 1985). Yoked $\mathrm{M}$ and $\mathrm{S}$ patterns had identical frequency distributions of IOIs. Also, structural grouping properties were controlled: The $\mathrm{M}$ and $\mathrm{S}$ pattern pairs had approximately the same number of tone groups of each size (one, two, or three tones).

The FP variable determined the final IOI of each sequences. Equally often, $\mathrm{M}$ and $\mathrm{S}$ patterns ended with IOIs of 250, 500, and 1,000 msec. Including the FP, M and $\mathrm{S}$ sets had identical means and standard deviations $(S D)$ of IOIs $(M=666.67 \mathrm{msec}, S D=302.77)$. Eighteen $\mathrm{M}$ and $\mathrm{S}$ rhythm-plus-FP sequences [3 (pattern) $\times 3$ (induction tone) $\times 2$ (target)] were created. All of the sequences had the same duration $(4,040 \mathrm{msec})$.

Design and Procedure. In Block 1, all of the subjects received only two-tone FP intervals (FPonly). In Block 2, the subjects were randomly assigned to one of two rhythm groups (M, S). Each group received rhythms (M or S) specifically linked to one of three FPs $(250,500,1,000 \mathrm{msec})$. Different rhythms plus FPs occurred ran- 
domly over trials. In all three conditions (FPonly, M, S), we used the same uniform distribution of FPs. Each trial block contained 54 trials. Intertrial intervals varied randomly between 2,750 and 3,250 $\mathrm{msec}(M=3,000 \mathrm{msec})$.

For each sequence, the subjects were told to judge whether a target pitch was lower or higher than that of the preceding tones as quickly and accurately as possible. The subjects pressed the "X" key on a keyboard with the pointer finger of their left hand for lower and the "M" key with the pointer finger of their right hand for higher. Responses were recorded within a 1,500-msec window from offset of the final tone.

\section{Results}

Accuracy and RT data were analyzed using identical 2 (group) $\times 3(\mathrm{FP})$ mixed-factorial ANOVAs, with group $(\mathrm{M}, \mathrm{S})$ as the between-subjects factor. Separate ANOVAs were performed on the Block 1 and Block 2 data. To assess bias, the proportion of higher responses, $P(H)$, was analyzed in each condition using the same ANOVA design. Significant effects are accompanied by partial eta squared $\left(\eta_{\mathrm{p}}^{2}\right)$ values to indicate the proportion of variance due to this effect in terms of its sums of squares: $\mathrm{SS}_{\text {effect }} /$ $\left(\mathrm{SS}_{\text {effect }}+\mathrm{SS}_{\text {error }}\right)$ (Keppel \& Wickens, 2004).

Accuracy. Accuracy was indexed as proportion correct $(\mathrm{PC})$. Accuracy in Block 1 (FPonly condition) is shown in Figure $3 \mathrm{~A}$. A main effect of FP on accuracy was found $\left[F(2,60)=7.18, p<.001, \eta_{\mathrm{p}}^{2}=.193\right]$, with strongly linear $\left[F(1,30)=12.97, p=.001, \eta_{\mathrm{p}}^{2}=.302\right]$ and weakly quadratic $\left[F(1,30)=3.58, p=.068, \eta_{\mathrm{p}}^{2}=\right.$ .107] trend components. Accuracy was higher at the 500$\operatorname{msec}(\mathrm{PC}=.777)$ and $1,000-\mathrm{msec}(\mathrm{PC}=.771) \mathrm{FPs}$ and lower at the $250-\mathrm{msec}$ FP $(\mathrm{PC}=.695)$. This accuracy profile is consistent with previous findings using the
variable-FP paradigm (Klein \& Kerr, 1974). Because of the random subject assignments to groups, no significant effects for group (main effect or trend components) were expected, and none were found $(p \mathrm{~s}>.642)$. An analysis of bias, $P(H)$, revealed a marginally significant FP effect $\left[F(2,60)=3.13, p=.051, \eta_{\mathrm{p}}^{2}=.094\right] ; P(H)$ decreased slightly as FP increased $(.510, .511$, and .478, respectively). Collapsed over FP, however, $P(H)$ was .50 . Together, these data rule out response bias as a factor in Block 1.

Accuracy in Block 2 (M or S condition) appears in Figure $3 \mathrm{~B}$. There was a marginally significant main effect of $\mathrm{FP}\left[F(2,60)=2.94, p=.06, \eta_{\mathrm{p}}^{2}=.089\right]$ and a negative linear trend $\left[F(1,30)=6.30, p=.012, \eta_{\mathrm{p}}^{2}=.173\right]$ : Accuracy was highest for the 250-msec FP and lowest for the 1,000-msec FP. Group (M, S) yielded neither a main effect nor an interaction with FP $(p \mathrm{~s}>.675)$. An analysis of $P(H)$ revealed no effects of rhythm, FP, or their interaction $(p$ s $>.740)$. Overall, mean $P(H)$ was .504. Finally, overall, accuracy in Block $2(M=.862, S D=.122)$ was higher than that in Block $1(M=.748, S D=.139)$. This difference can be attributed to several causes (e.g., pitch, rhythmic context, practice), which cannot be addressed by the present design.

RTs. A preliminary RT analysis revealed longer error RTs $(M=811 \mathrm{msec})$ than correct RTs $(M=667 \mathrm{msec})$. We analyzed only the latter (removing $19.5 \%$ of the data). Because RT distributions are positively skewed (ex-Gaussian; e.g., Luce, 1986; Ratcliff, 1993), a 2.5-SD cutoff applied to each subject's correct RT data, eliminating an additional $1.8 \%$ of responses. Individual mean RTs (collapsed over FPs) ranged from 487 to $835 \mathrm{msec}$
A Block 1

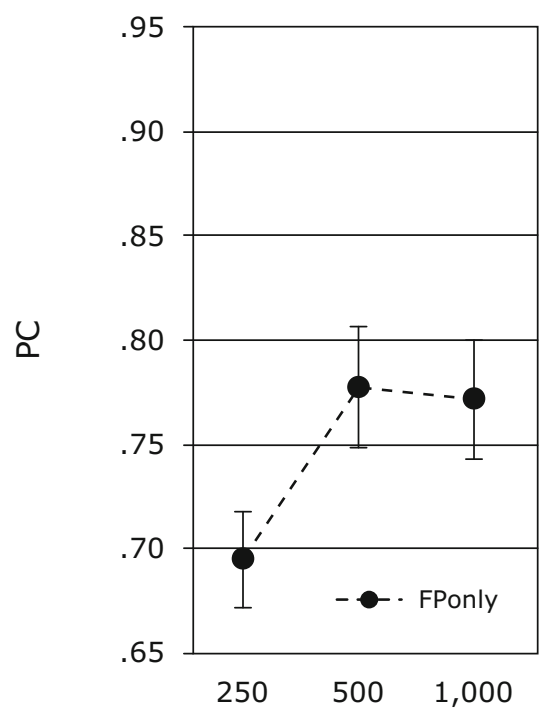

B Block 2

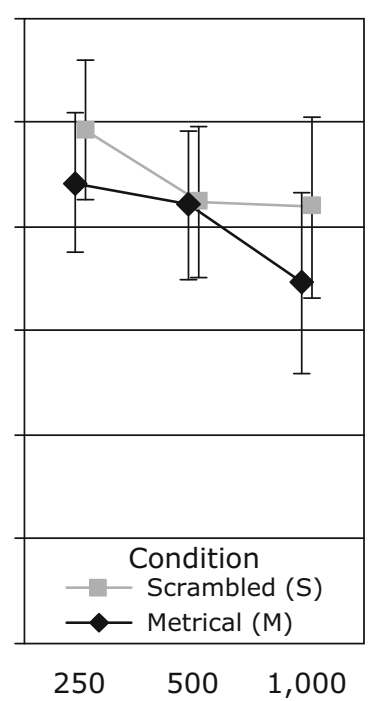

Foreperiod (msec)

Figure 3. Experiment 1. (A) Mean proportion correct (PC) in Block 1 as a function of foreperiod (FP) in the FPonly condition. (B) Mean PC in Block 2 as a function of $\mathrm{FP}$ and group $(\mathrm{M}, \mathrm{S})$. 


\section{A Block 1}

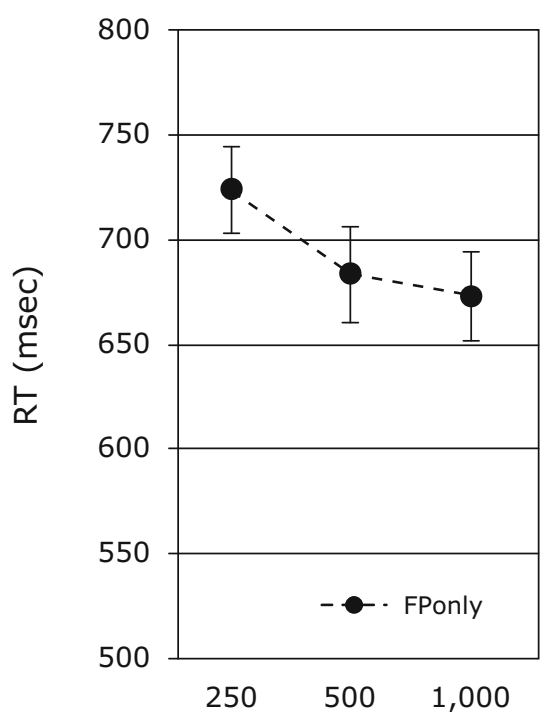

\section{B Block 2}

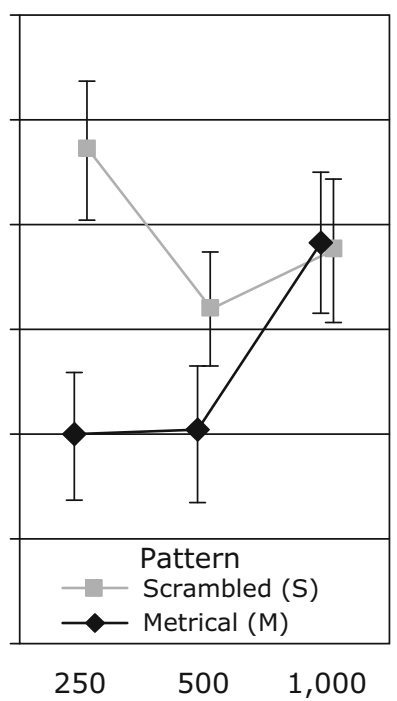

Foreperiod (msec)

Figure 4. Experiment 1. (A) Mean reaction time (RT) in Block 1 as a function of foreperiod (FP) in the FPonly condition. (B) Mean RT in Block 2 as a function of FP and group $(\mathrm{M}, \mathrm{S})$.

(group $M=682, S D=98$ ). Figure 4 presents mean RTs for Experiment 1.

Block 1 (FPonly condition) RTs in the FPonly condition appear in Figure 4A. The main effect of FP effect was significant $[F(2,60)=3.42, p<.05]$; mean RT decreased as FP increased, leading to a significant negative linear trend $\left[F(1,30)=5.55, p=.025, \eta_{\mathrm{p}}^{2}=.156\right]$. Group assignment had no effect on performance.

Block 2 (M, S) RTs appear in Figure 4B. Overall, the RTs of subjects in the M group were notably faster $(M=$ $631 \mathrm{msec})$ than the RTs of those in the $\mathrm{S}$ group $(M=$ $694 \mathrm{msec}$ ). This main effect did not reach statistical significance because of a strong group $\times$ FP interaction and intersubject variability in RTs (but see the Speed-accuracy trade-offs section below). There was a significant main effect of FP $\left[F(2,60)=8.91, p<.001, \eta_{\mathrm{p}}^{2}=.229\right]$ and a significant quadratic trend $[F(1,30)=15.45, p<.001$, $\left.\eta_{\mathrm{p}}^{2}=.340\right]$, most evident in the $\mathrm{S}$ group.

A robust group $\times$ FP interaction $[F(2,60)=12.24, p<$ $\left..001, \eta_{\mathrm{p}}^{2}=.290\right]$ was characterized by a group $\times$ linear FP interaction $\left[F(1,30)=24.42, p<.001, \eta_{\mathrm{p}}^{2}=.449\right]$ but no group $\times$ quadratic FP interaction $(F<.25)$. Specifically, a positive linear trend occurred with the $\mathrm{M}$ group $\left[F(1,30)=20.95, p<.001, \eta_{\mathrm{p}}^{2}=.41\right]$ and a negative linear trend occurred with the $\mathrm{S}$ group $[F(1,30)=5.81$, $\left.p=.022, \eta_{\mathrm{p}}^{2}=.162\right]$. Relative to the $1,000-\mathrm{msec}$ FP, RTs to the $250-\mathrm{msec} \mathrm{FP}$ were faster for M patterns and slower for $\mathrm{S}$ patterns.

Speed-accuracy trade-offs. Finally, we assessed speed-accuracy trade-offs. Spearman correlations between average RT and PC were negative for all 32 subjects in Block $1(\rho=-.262, p=.147)$, Block 2's M group ( $\rho=$
$-.585, p=.017)$, and Block 2's S group ( $\rho=-.414$, $p=.111)$. Higher accuracy was associated with faster (rather than slower) responding, providing no support for a speed-accuracy trade-off. Furthermore, inspection of speed-accuracy scatterplots revealed a particularly poor $\mathrm{M}$ subject $(\mathrm{PC}=.544)$. Removal of this subject and the corresponding poorest-performing S subject $(\mathrm{PC}=.643)$ yielded a significant main effect of group $[F(1,28)=5.14$, $\left.p=.031, \eta_{\mathrm{p}}^{2}=.155\right]$. Adjusted mean RTs were 609 and $691 \mathrm{msec}$ for the $\mathrm{M}$ and $\mathrm{S}$ groups, respectively.

\section{Discussion}

A classic variable-FP effect (a decreasing RT-FP profile) was observed in the FPonly condition and also in the $\mathrm{S}$ condition of Block 2. These RT profiles are consistent with the probabilistic uncertainty hypothesis. They also confirm that the present modification of the variable-FP paradigm yields results similar to those of the original paradigm.

Other findings are more consistent with dynamic attending theory. First, the RTs in Block 2 were generally shorter to targets ending $\mathrm{M}$ patterns than to targets ending S patterns, especially with shorter FPs. Second, rhythmic context modulated the RT-FP profile. Two opposing linear trends were found: a negative RT-FP profile for the S group (which is common in variable-FP paradigms) and a positive RT-FP profile for the $\mathrm{M}$ group (common in constant-FP paradigms). This interaction is consistent with the DAT prediction that metrical constraints will sensitize listeners to implied periodicities congruent with final FPs. Thus, M listeners exhibited shorter RTs to shorter FPs, in contrast to their performance in the FPonly condition of 
Block 1. Such a shift did not happen for the S group. It appears that the $\mathrm{M}$ and $\mathrm{S}$ groups relied on different aspects of these global contexts to prepare their responses.

One aspect of performance in the $\mathrm{S}$ group in Block 2 that did not resemble baseline performance was the quadratic trend component in the RT-FP profile. This U-shaped profile showed relatively longer RTs to the longest FP. ${ }^{3}$ As was noted in the introduction, such a profile is not uncommon in variable-FP paradigms; its appearance is typically attributed to a drop in uncertainty with respect to the longest FP in a set and a concomitant scaling of time estimation error with this (predictable) time interval (Klemmer, 1956).

Three other features of the present paradigm may also have factored into the lengthening of RTs to the 1,000msec FP in S group. First, the baseline stimuli may have successfully familiarized the subjects with the inventory of FPs. Second, some (unknown) structural feature of the $\mathrm{S}$ patterns may have permitted the listeners to anticipate the occurrence of the 1,000-msec FP. Decreased uncertainty about what for the 1,000 -msec FP in the S condition (as well as in the M condition) would lead to a scaling of time estimation error with FP resulting in longer RTs. Third, difficulty with the pitch judgment task may have increased with FP in both rhythmic conditions, because of memory limitations, leading to slower responses at the longest FP. Indeed, accuracy dropped significantly for the longest FP. Importantly, all three of these features would suggest that RTs to the longest FP should be similar in the FPonly, M, and S conditions, and in fact, they were (673, 688, and $690 \mathrm{msec}$, respectively). In Experiment 2, we evaluated these explanations using a simpler task.

Finally, two other features of the Experiment 1 data should be mentioned. First, with respect to the functional FP interval in this task, it might be argued that the total sequence duration in Block 2 functions as the FP and not the sequence-final time interval. Because the total duration was constant across all sequences, however, it does not explain the distinctive RT profiles observed.

Second, null accuracy effects were found in Block 2. It has been reported in several previous studies that rhythmic context improves target identification at temporally expected versus unexpected points in time (e.g., Jones, Johnston, \& Puente, 2006; Jones, Kidd, \& Wetzel, 1981; Kidd, Boltz, \& Jones, 1984; Lange \& Heil, 2008). The present design differs from those mentioned above, however. Earlier designs relied on inducing strong, patternspecific expectancies that could be violated by early or late target timing. In the present design, a target tone always occurred on time.

Given the clear effect of rhythmic structure on RTs to FPs, in Experiment 2, we sought to further explore how changes in this global context across blocks affect RT-FP profiles.

\section{EXPERIMENT 2}

In Experiment 2, we pursued the robustness of global context effects on RTs to sequence-final FPs. It differed from Experiment 1 in four ways.
First, the task was changed from a more difficult pitchdiscrimination task (yoked target tones at \pm 1 semitone from the standard) to a simpler pitch-identification task (two target tones with fixed pitches).

Second, the initial baseline condition was eliminated. This permits a clearer assessment of true differences between $\mathrm{M}$ and $\mathrm{S}$ rhythms. It also allows assessment of determinants of the quadratic RT profile for S patterns observed in Experiment 1. One explanation of that trend placed the explanatory burden on carryover effects from Block 1, whereas another placed this burden on the S pattern structure itself in Block 2. In Experiment 2, different groups of listeners initially received a block of either M or $\mathrm{S}$ patterns. If the $\mathrm{S}$ pattern structure itself determines a quadratic profile of RTs over FPs, the trend observed in Experiment 1 (Block 2) should be replicated with $\mathrm{S}$ rhythms in Block 1 of Experiment 2. But if the quadratic trend resulted from carryover effects from the FPonly trials, it would not appear in RTs to S patterns in Block 1.

Third, carryover effects were assessed. We introduced a counterbalance variable; listeners receiving $\mathrm{M}$ rhythms in Block 1 received S rhythms in Block 2 and vice versa. If carryover effects are absent and global context effects are robust, similar RT-FP profiles should emerge for the $\mathrm{M}$ and $\mathrm{S}$ patterns in Block 1 and Block 2.

Fourth, all six patterns in each rhythm category were utilized. This was designed to discourage a counting strategy, since the number of tones per sequence was not constant. It also introduced a categorical association in that two different precursor rhythms were linked with the same FP. Unlike the S patterns, all of the $\mathrm{M}$ patterns were categorically related by the same $4 / 4$ meter; they share the same putatively induced periods $(250,500$, and $1,000 \mathrm{msec})$ linked by consonant relationships (i.e., period ratios of $1: 1,1: 2$, and $1: 4)$. If listeners rely on such relational metrical invariants, they should respond to the $\mathrm{M}$ patterns much as listeners in Experiment 1 did.

\section{Method}

Subjects. Thirty-six introductory psychology students from The Ohio State University served as subjects in return for course credit. All of them reported normal hearing and less than 5 years of musical training. The subjects were run in groups of 1-4 subjects and were randomly assigned to either an MS (M then S) condition or an SM (S then M) condition (both $n \mathrm{~s}=18$ ).

Apparatus. The stimuli were generated with MIDILAB Version 6.0 software (Todd, Boltz, \& Jones, 1989) interfaced with a Yamaha TG100 Tone Generator (sine voice) and presented binaurally over Beyerdynamic DT 770 headphones at a comfortable listening level. Responses (accuracy, RT) were automatically recorded by the MIDILAB software.

Stimuli and Materials. All six sequences of each rhythmic category $(\mathrm{M}, \mathrm{S})$ shown in Figure 1 were used. The induction sequences contained six or seven repeated tones with a pitch of $F_{\sharp 5}$ (740 Hz; $10 \mathrm{msec}$, with 2-msec rise/decay times) followed by a target tone ( $25 \mathrm{msec}$, with 5 -msec rise/decay times). Using a simpler pitch-judgment task, the target assumed one of two distinctively different pitches (equally often), each six semitones distant from the induction tone: C5 (523.3 Hz; low) or C6 (1046.4 Hz; high). As in Experiment 1, all sequences had a duration of $4 \mathrm{sec}$ between the onset of the initial tone and the onset of target. There was a total of $2($ pattern $) \times 2$ (sequence length $) \times 3(\mathrm{FP}) \times 2($ target $)=24$ unique 
patterns, $12 \mathrm{M}$ and $12 \mathrm{~S}$. The $\mathrm{M}$ and $\mathrm{S}$ patterns, as a set, had identical IOI frequency distributions. The statistical IOI properties of the $\mathrm{M}$ and $\mathrm{S}$ patterns were equated for these eight-tone sequences $(M=$ $571.43 \mathrm{msec}, S D=345.03 \mathrm{msec}$ ).

Design and Conditions. The design again crossed pattern (M, S) with FP $(250,500,1,000 \mathrm{msec})$ and counterbalance order (MS, SM) Half of the subjects heard the M block first (MS order); the remainder heard the S block first (SM order). Within each M and S block, the same uniform distribution of FP values appeared over trials.

Procedure. The task required a pitch-identification judgment (low or high) via two labeled buttons within 1,500 msec of the onset of the target. The instructions were identical to those of Experiment 1 . The intertrial interval was $3,000 \mathrm{msec}$ (onset of the target tone to onset of the next pattern). The subjects received two trial blocks of 72 trials, with either the $\mathrm{M}$ or the $\mathrm{S}$ block first. Each unique pattern was presented six times per block.

Finally, the listeners completed a four-question survey to ascertain whether they noticed any differences between the M and S patterns, found the M or S pattern more difficult, counted tones, or timed their responses to match a rhythm.

\section{Results}

PC and RT data were analyzed using a $2 \times 2 \times 3$ mixed factorial ANOVA, with one between-subjects factororder (MS, SM) - and two within-subjects factorspattern $(\mathrm{M}, \mathrm{S})$ and $\mathrm{FP}(250,500,1,000 \mathrm{msec})$.

Accuracy. Overall PC was very high $(M=.976$, $S D=.025)$. The ANOVA yielded no significant effects for pattern, order, or FP and no significant interaction (all $F \mathrm{~s}<1)$. The $P(H)$ analysis similarly revealed no significant effects; overall $P(H)$ was .504. Finally, no evidence of speed-accuracy trade-offs was present.

RTs. Error trials were excluded from the analysis $(2.7 \%$ of data). As in Experiment 1, a 2.5-SD cutoff was applied to each subject's correct RTs, eliminating an additional $3.4 \%$ of the data. Mean RTs ranged from 245 to $743 \mathrm{msec}$ (grand mean $=391 \mathrm{msec}, S D=117$ ).

Figures $5 \mathrm{~A}$ and $5 \mathrm{~B}$ plot the mean $\mathrm{RT}$ data as a function of pattern, FP, and block $(1,2)$. The counterbalancing of pattern (MS, SM) meant that the subjects who received the M patterns in Block 1 received the S patterns in Block 2 and vice versa (in Figures 5A and 5B, solid and dashed lines denote, respectively, MS and SM order). A preliminary $2 \times$ $2 \times 3$ mixed factorial ANOVA crossed order (MS, SM) with pattern (M, S) and FP $(250,500,1,000 \mathrm{msec})$. There was a significant main effect of pattern $[F(1,34)=6.56$, $\left.p=.015, \eta_{\mathrm{p}}^{2}=.162\right]$. Overall, RTs to M patterns $(M=$ $374 \mathrm{msec})$ were shorter than those to $\mathrm{S}$ patterns $(M=$ $391 \mathrm{msec}$ ); that is, the solid black line points show shorter RTs than the solid gray line points and the dashed black line points show shorter RTs than the dashed gray line points. A significant pattern $\times \mathrm{FP}$ interaction $[F(2,68)=9.75, p<$ $\left..001, \eta_{\mathrm{p}}^{2}=.223\right]$ and a pattern $\times \mathrm{FP} \times$ order interaction $\left[F(2,68)=5.22, p<.005, \eta_{\mathrm{p}}^{2}=.133\right]$ were both present.

To clarify the effects of rhythmic context in Block 1 and Block 2, we performed separate ANOVAs on the data from each block. The clearest effects of rhythm and FP appear in Block 1. Figure 5A shows mean RT as function of these two factors. The listeners receiving the M patterns responded significantly faster than the listeners receiving the $\mathrm{S}$ patterns $\left[F(1,34)=4.46, p<.05, \eta_{\mathrm{p}}^{2}=.116\right]$. A trend analysis revealed a significant linear trend over FPs for the M patterns, $\left[F(1,34)=11.54, p<.005, \eta_{\mathrm{p}}^{2}=\right.$ $.253]$; there were no significant linear $(p=.480)$ or quadratic $(p=.099)$ trends for the $\mathrm{S}$ patterns.

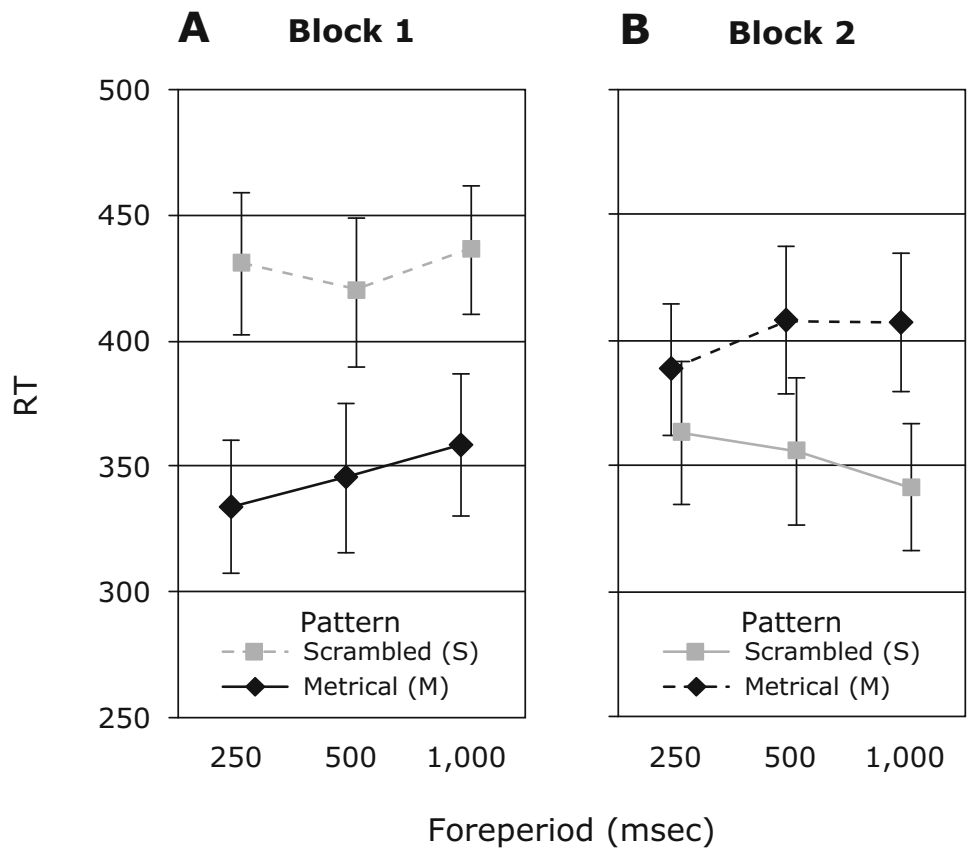

Figure 5. Experiment 2. (A) Mean reaction time (RT) in Block 1 as a function of pattern (M, S) and foreperiod (FP). (B) Mean RT in Block 2 as a function of pattern and FP. In both panels, counterbalance order MS (M then $S$ ) is highlighted with solid lines; SM (S then M) is highlighted with dashed lines. 
In Block 2 (Figure 5B), there was no main effect of pattern $(p>.30)$; instead, pattern interacted with FP $\left[F(2,68)=6.11, p<.001, \eta_{\mathrm{p}}^{2}=.152\right]$. This interaction was characterized by two opposing linear trends. RTs to M patterns increased with FP $[F(1,34)=5.05, p<.05$, $\left.\eta_{\mathrm{p}}^{2}=.176\right]$, whereas RTs to S patterns decreased with FP $\left[F(1,34)=7.24, p<.025, \eta_{\mathrm{p}}^{2}=.176\right]$. The interaction of these opposing linear trends was significant $[F(1,34)=$ $\left.12.19, p<.001, \eta_{\mathrm{p}}^{2}=.264\right]$.

Questionnaire results. The tallies of 36 respondents to the questions are as follows: noticed differences between $\mathrm{M}$ and $\mathrm{S}, 1$ no, 2 unsure, and 33 yes; pattern difficulty, $25 \mathrm{M}$ easier, 11 no difference, $0 \mathrm{~S}$ easier; counted tones, 14 never, 17 sometimes, 5 always; timed responses to match a rhythm, 6 never, 8 unsure, 20 depended on the rhythm, 2 always.

\section{Discussion}

The listeners receiving $\mathrm{M}$ patterns responded more rapidly to target tones than those receiving S ones in Block 1. In addition, rhythmic context modulated the RT-FP profiles in both trial blocks. Consistent with these findings, most subjects reported noticing differences between the M and S patterns $(92 \%)$, with a significant majority $(69 \%)$ reporting the former as easier.

Many subjects had difficulty responding to the S patterns; indeed no subjects reported finding the $\mathrm{S}$ patterns easier than the M patterns. This difficulty was reflected in Block 1 RTs, where S patterns elicited overall long RTs and an almost flat RT-FP profile. Such a profile suggests that the listeners had persistently high uncertainty levels about FP identities and/or set size, consistent with the probabilistic uncertainty hypothesis outlined in the introduction. For different reasons, DAT also predicts relatively slow responding to $\mathrm{S}$ patterns and a flat RT-FP profile. In this view, the lack of rhythmic coherence in the $\mathrm{S}$ patterns prevented any FP-related preparatory activity.

The most straightforward picture of global context effects, absent carryover effects, appears in the Block 1 data. These findings clarify the $\mathrm{M}$ versus $\mathrm{S}$ influences on RTs; they also (in conjunction with Experiment 1) confirm the presence of important carryover effects in FP paradigms. That is, in Block 1 of Experiment 2, all traces of a quadratic trend in responding to $S$ patterns vanished. Because RTs to the S patterns lengthened for all FPs instead of shortening for the longest FP, it is unlikely that the quadratic trend of Experiment 1 resulted from task difficulty due to limited memory for the induction pitch. Instead, we infer that the U-shaped trend in Experiment 1's Block 2 resulted from baseline carryover effects. It is likely that the quadratic RT-FP profile for the S patterns in Experiment 1 resulted from a carryover of FP set size knowledge acquired from the preceding FPonly condition.

Most revealing is the difference in RTs between the M and $\mathrm{S}$ patterns in Block 1. Whereas the listeners receiving $\mathrm{S}$ patterns showed long RTs that were unmodulated by FPs, the listeners receiving M patterns responded rapidly and showed an ascending RT-FP profile characteristic of constant-FP paradigms. The latter profile implies that the
M listeners had no uncertainty about the what of FPs; instead, they seemed to try to match their RTs to the FPs (a point developed in the General Discussion section). Most importantly, the increasing RT-FP profile with the M patterns occurred in a variable-FP design with a global context that is statistically identical to the context provided by the $\mathrm{S}$ patterns.

The Block 2 data are more complicated to interpret because of carryover effects. Nevertheless, they shed light on the robustness of global context effects. Specifically, distinctive RT-FP profiles for the $\mathrm{M}$ and $\mathrm{S}$ patterns were pronounced in Block 2. Although both subject groups (SM and MS) had to accommodate a changed rhythm in Block 2, it is telling that they did so in a manner that yielded the classic decreasing RT-FP profile to $\mathrm{S}$ patterns and the classic increasing RT-FP profile to M patterns.

These data also confirm carryover effects in blocked designs and the presence of set familiarization. The data from the listeners with the S patterns in Block 1 (the SM group) indicate that the $\mathrm{S}$ patterns alone did not familiarize the listeners with FP intervals. Rather, the observed flat RT-FP profile implies that the listeners did not gain familiarity with the FP set. By contrast, the listeners initially exposed to the M patterns (the MS group) showed an RT-FP profile in Block 1 that suggests familiarity with FPs. Confirming this interpretation, the MS listeners exhibited a decreasing RT-FP profile to the S patterns in Block 2 (i.e., they did not exhibit a flat RT profile). In summary, the most parsimonious explanation of these carryover effects holds that in Block 1, the M patterns (and FPonly stimuli) were effective in familiarizing the listeners with the what of an FP set, but the S patterns were not.

Finally, the absence of a main effect of rhythm in Block 2 is due to a surprising carryover effect that is best described as $R T$ perseveration. In both counterbalance orders, the average RTs of the different subject groups persisted from Block 1 into Block 2, thereby eliminating a rhythm main effect in Block 2. That is, in Block 2, the RTs of the SM group to the M patterns were long (relative to those to the $\mathrm{M}$ patterns in Block 1), presumably perseverating long RTs to the S patterns in Block 1; similarly, RTs of the MS group to the $\mathrm{S}$ patterns were relatively short (relative to the S patterns in Block 1). Bertelson (1967) reported a somewhat similar carryover effect between control and experimental FP conditions. He offered no explanation, nor do we. Yet, these various carryover effects suggest that caution is warranted during the interpretation of RTs in blocked designs.

\section{GENERAL DISCUSSION}

A coherent rhythmic context both speeds overall responding and systematically modulates choice RTs to different sequence-final time intervals. Although these findings are consistent with predictions of dynamic attending theory, they also provide partial support for the probabilistic uncertainty hypothesis. Specifically, the latter hypothesis nicely explains RT profiles in the isolated 
FP condition (Experiment 1, Block 1) and conditions in which statistical descriptions of global rhythmic structure may be more appropriate (e.g., S rhythms). The next two sections discuss the implications of global context for understanding temporal preparation and interactions with FP intervals. In the final section, we consider questions raised by the present findings.

\section{Global Context and Temporal Preparation}

The probabilistic uncertainty hypothesis maintains that as statistical uncertainty is reduced in global FP contexts, preparation increases and RTs decrease. We tested this hypothesis by equating the frequency distribution of time intervals in two relationally different global contexts using the same variable-FP paradigm. This uncertainty hypothesis predicts that RTs to sequence-final FP intervals should not differ as a function of these two contexts. However, as was most apparent in Block 1 of Experiment 2, overall RTs were shorter to patterns with metrical time relationships than to those with no metrical time relationships. If we retain the assumption that shorter RTs signify improved temporal preparation, this main effect of rhythmic context implies that preparation for forthcoming time intervals in extended temporal contexts is not entirely determined by probabilistic uncertainties associated with frequency distributions of unrelated time intervals.

We suggest instead that temporal preparation is influenced by the presence of salient temporal relationships. In pinpointing these relationships, we can rule out explanations of rhythmical coherence on the basis of Gestalt principles of tone groups or various statistical properties, which were comparable for both $\mathrm{M}$ and $\mathrm{S}$ rhythmic categories. Instead, rhythmic coherence is best expressed here in terms of metrical relationships among accented time levels. Two approaches that differentiate the $\mathrm{M}$ and $\mathrm{S}$ contexts are the coding theory of Povel and Essens (1985) and dynamic attending theory.

Povel and Essens's (1985) memory code approach has been highly influential in explaining rhythm perception. It assumes that the degree of conformance of accent locations in a temporal pattern to an induced clock grid drives memory encoding for that pattern. Here, accents in M patterns (but not $\mathrm{S}$ patterns) conformed to a 4/4 metrical grid, leading to more economical memory codes for $\mathrm{M}$ than for S patterns (see note 2). Under the assumption (not made explicit by Povel \& Essens) that a more economical memory code insures shorter choice RTs, the main effect of rhythm observed in the present study converges with data from other tasks (reproduction, synchronization) to support this coding model (Grahn \& Brett, 2009; McAuley, 2010; McAuley \& Semple, 1999; Patel, Iversen, Chen, \& Repp, 2005; Povel \& Essens, 1985).

Importantly, the Povel and Essens (1985) model does not directly address online temporal preparation; instead, its predictions rest on a retrospective encoding of already finished sequences. By contrast, DAT specifically links an internal preparatory activity to in-the-moment attending. This activity involves early entrainment of attending oscillations that lock into accented time spans as a se- quence unfolds (Large \& Jones, 1999; Pfordresher, 2003). That certain rhythmic contexts conform to this entrainment scenario and shorten choice RTs has been shown by Martin et al. (2005). In the present set of rhythms, early emergence of preparatory activity involves temporal anticipations, leading to shorter RTs to M than to S patterns. The present data converge with those of other studies reporting shorter RTs to simple than to complex M patterns (Ellis \& Jones, 2009) and better temporal resolution with M than with S rhythms (Jones \& Yee, 1997; Large \& Jones, 1999).

Although the probabilistic uncertainty hypothesis does not address the impact of rhythmic coherence on the timing of preparatory activities, conversely DAT does not address probabilistic uncertainties about the inventory of what FPs will occur. Thus, the probabilistic uncertainty hypothesis, but not DAT, correctly predicts decreasing RT-FP profiles of certain S conditions.

Nevertheless, temporal preparation is a construct shared by approaches to both rhythm and FPs. Our finding that listeners respond faster to $M$ than to $S$ patterns implies that certain perceived regularities in $\mathrm{M}$ patterns hastened preparation. A common issue in FP research concerns whether preparatory activities begin early (i.e., are perceptual) or late (i.e., are motor) (see Müller-Gethmann et al., 2003, for an excellent review). Some maintain that nonspecific temporal preparation in constant-FP paradigms (e.g., for short FPs) begins as early as the onset of a warning tone in designs with a single warning tone (Los \& Schut, 2008). The present findings imply that the introduction of rhythmic structure may modulate this effect. If a warning tone (i.e., the onset of an FP) is preceded by a metrical (vs. a nonmetrical) rhythmic context, DAT predicts that temporal anticipations will emerge significantly earlier than the warning tone itself. Moreover, in the present study, no sequence tone (except the target) required an overt motor response. This suggests that stimulus rhythm alone engages perceptual/attentional activities early in metrical sequences. Related EEG (e.g., Praamstra, Kourtis, Kwok, \& Oostenveld, 2006; Schmidt-Kassow, Schubotz, \& Kotz, 2009) and fMRI (Grahn \& Brett, 2007) evidence supports this: Metrical (vs. nonmetrical) patterns engage both perceptual and motor brain areas (e.g., basal ganglia, supplementary motor area).

\section{Rhythmic Context Modulates \\ Responses to FP Intervals}

The most notable finding from this study is the differential impact of M and S contexts on RTs to FPs. This interaction was manifest as a decreasing (or flat) RT-FP profile with S patterns and an increasing RT-FP profile with $\mathrm{M}$ patterns. A strictly statistical analysis of global context provides no reason to expect this interaction, since the $\mathrm{M}$ and $\mathrm{S}$ pattern sets were matched in terms of number and distributions of IOIs, and both conditions utilized a variable-FP design. What other aspects of the present paradigm might account for these differences? We briefly consider probabilistic uncertainty, target cuing, and FP priming. 
First, the probabilistic uncertainty hypothesis correctly predicts RT-FP profiles for S patterns: a flat RT-FP profile when the set of FPs is unclear (i.e., S patterns heard in Block 1) and a decreasing RT-FP profile when the set of FPs is clear (i.e., S patterns in Block 2 following $\mathrm{M}$ patterns in Block 1). However, the probabilistic uncertainty hypothesis does not predict that metrical constraints (in M patterns) will reverse the RT-FP profile, making it resemble the RT-FP profile found with a constantFP paradigm. In fact, ascending RT-FP profiles in this variable-FP paradigm present a puzzle for the probabilistic uncertainty hypothesis.

Second, the interaction of rhythmic context with FP may result from differences in target cuing effectiveness between $\mathrm{M}$ and $\mathrm{S}$ patterns. If the penultimate tone of an $\mathrm{M}$ pattern functioned as a distinctive warning cue, listeners may have learned probabilistic temporal contingencies between the cue and the target. If so, the penultimate tone would serve as a valid endogenous cue for learning different FPs (Coull \& Nobre, 1998). However, in the present design, we used a nonspecific FP task: The pitch and duration of all induction tones were identical, meaning that the penultimate tone provided no information about a target. Also, the frequency of associating this cue with a given FP was identical for the $\mathrm{M}$ and $\mathrm{S}$ patterns. In short, the penultimate tone cannot function as a distinctive cue for endogenous attending, as was discussed by Coull and Nobre (1998; see also Correa, Lupiáñez, \& Tudela, 2006; Coull, Frith, Büchel, \& Nobre, 2000). Cuing may ultimately prove an important component in this story, but it does not explain our interaction of rhythmic context with FP.

Third, FPs might have been temporally primed. If a precursor pattern attached to a particular FP contained more IOIs equal to that FP, this might prime that FP. However, because the $\mathrm{M}$ and $\mathrm{S}$ patterns comprised the same set of IOIs, priming in this sense fails to explain the RT differences. Similarly, whereas models such as Povel and Essens's (1985) offer increased explanatory power by considering the relative timing of metrical relations (rather than a purely statistical description of IOIs), encoding models do not predict an interaction of rhythm with FP, because no significant correlation exists between the encoding strength of Figure 1 patterns (either M or S) and associated FPs (see note 2).

\section{Metric Binding, Oscillator Variability, and RTs}

As was noted earlier, it is puzzling that the variable-FP paradigm with $\mathrm{M}$ patterns yielded a profile more typically observed in a constant-FP design. One resolution of this puzzle holds that the presentation of a set of M patterns within a variable-FP paradigm functions as if the same FP were repeated over trials. As a result, subjective uncertainty about the what of the FPs is minimized for each of the three FPs ending M patterns. Thus, the positive linear RT trend with FPs in the M patterns reflects only increasing uncertainty about when FPs will end, much as in constant-FP paradigms. Why does this happen for M patterns but not $\mathrm{S}$ patterns? DAT provides a possible answer.
As was discussed in the introduction, DAT addresses the interaction of rhythmic structure and FP via the concept of congruence between a context-induced beat period and a final FP. ${ }^{4}$ Maximal congruence refers to a 1:1 matching of an oscillator period with a stimulus IOI. Thus, each of the three oscillations depicted in Figure 2 has a period $(250,500$, or $1,000 \mathrm{msec})$ that is maximally congruent with one FP $(250,500$, or $1,000 \mathrm{msec})$. These oscillations are simultaneously induced by invariant aspects of metrical contexts, as is proposed by the metric binding hypothesis (Jones, 2009). Moreover, they are hypothesized to bind together into an internalized metric hierarchy that is common to the whole category of M patterns. As a set, these oscillations prepare listeners for future congruent time intervals in a sequence, including the final interval. For example, the FP in pattern M1 is maximally congruent with the oscillation with a $250-\mathrm{msec}$ period. Similarly, the FP of the M2 pattern is maximally congruent with the oscillation with a 500 -msec period. ${ }^{5}$

Crucially, the mechanism for predicting RTs to a given FP in metrical contexts depends on oscillator variability. Simply put, the RT to an FP is a function of the variability of the oscillator matched to that FP. In entrainment theory, oscillator variability is scaled to the period of an active oscillator: Longer oscillator periods elicit proportionately greater temporal variability. Therefore, since RTs reflect temporal variability, they should increase with variability of the oscillation congruent with a given FP, consistent with Weber's law. As a result, DAT predicts that RTs to FPs of M patterns will positively covary with FP durations. In contrast, although the S patterns shared the same set of FPs as the $\mathrm{M}$ patterns, the $\mathrm{S}$ pattern structure elicited neither oscillations nor entrainment. Consequently, the ascending RT-FP profile elicited by the M patterns is predicted to be absent with the $\mathrm{S}$ patterns, and indeed it was.

From the perspective of DAT, the lengthening of RTs at longer FPs could instead be interpreted as reflecting listeners' attempts to match their RTs to the FPs. Our questionnaire indicated that 22 out of the 36 subjects in Experiment 2 were aware of their attempts to match their RTs to at least some pattern endings. If RTs to M patterns are determined (at least in part) by entrained oscillators, longer RTs reflect a dynamic attentional system at work rather than poorer performance in the task. This is an intriguing hypothesis that deserves further exploration.

Note an alternative hypothesis regarding metric binding. Since all three oscillator periods are active in M patterns, it might seem logical that the smallest period $(250 \mathrm{msec})$, with the least amount of variability, should be the one that is always monitored, leading to short RTs for all FPs. The data from the M patterns, however, argued against such a process.

To summarize, when a set of stimuli exhibits metric regularities (M patterns), a binding of internal oscillations forms temporal expectancies about specific FPs and leads to an ascending RT-FP profile. When metric regularities are absent, but a set of FPs is known (S patterns in Block 2), FP distribution properties elicit probabilistic expectations and lead to a descending RT-FP profile. When 
metric regularities are absent and a set of FPs is unknown (S patterns in Block 1), probabilistic uncertainty for all FPs is high and expectancies about FP are absent, leading to a flat RT-FP profile.

Finally, we return to the puzzle noted earlier concerning increasing RT-FP profiles in variable-FP M patterns that resemble profiles of constant-FP paradigms. On the surface, these two contexts differ dramatically: In constant-FP designs, the same FP is repeated trial after trial, whereas in our experiments, both FPs and M patterns differed from trial to trial. Yet our findings suggest that $\mathrm{M}$ patterns eliminate probabilistic uncertainties about the what of FPs (as do constant-FP paradigms), leaving only temporal uncertainty about when an FP ends. What appears to permit this is what remains constant over trials within an M block: an implied time hierarchy. Thus, one solution to this puzzle is that the invariance of an internalized metrical time hierarchy parallels the constancy of a single FP in the constant-FP paradigm.

\section{Conclusions and Future Directions}

The present findings contribute to research on temporal preparation and attending. Advantages of temporal preparation are found in time judgment/discrimination tasks using FP designs (Bausenhart, Rolke, \& Ulrich, 2008; Correa, Sanabria, Spence, Tudela, \& Lupiáñez, 2006; Grondin \& Rammsayer, 2003). In tasks in which rhythm implicitly drives temporal expectancies, accuracy for on-time targets increases (Correa \& Nobre, 2008; Jones, Boltz, \& Kidd, 1982; Martin et al., 2005; Olson \& Chun, 2001) and time judgment/discrimination improves (Barnes \& Jones, 2000; Large \& Jones, 1999; McAuley \& Jones, 2003; McAuley \& Kidd, 1998). A common explanatory construct is temporally guided attending.

A broader account of the determinants of preparatory activities considers how the nature of these activities varies with context. Expectancies (or conversely, uncertainties) about future time intervals may have a probabilistic origin in contexts with low rhythmic/temporal coherence (e.g., conventional variable-FP conditions, FPonly patterns, and S patterns) and a more deterministic (entrainment) origin in situations that afford high rhythmic/temporal coherence (e.g., single FPs in constant-FP conditions and M patterns in variable-FP conditions). Perhaps a continuum of temporal structure coherence exists, ranging from low to high, such that expectancy determinants shift from probabilistically driven to entrainment driven as coherence increases (Jones \& Boltz, 1989).

At least five issues are raised by the present data that warrant more study. First, what are the salient characteristics of nonisochronous rhythms that determine temporal coherence? Are other kinds of nonmetrical, but rhythmic, constraints similarly effective?

Second, what role does pattern-specific rhythm learning play in the observed data? Responses to M (vs. S) patterns may have relied on some form of perceptual learning with respect to individual rhythmic patterns (see note 4). If learning a pattern drives the ascending RT-FP profile, would increased exposure to $\mathrm{S}$ patterns then yield that profile? Little theory or research has addressed rhythm learning (but see Jones, 2009).

Third, what role does tempo play? Since rhythms are relative time patterns, would the RT-FP profile time scale with changes in tempo (e.g., McAuley \& Miller, 2007)? If so, is time scaling more precise for $M$ than for S patterns?

Fourth, what is the nature of carryover effects between blocks? For example, although RT-FP profiles themselves seem linked to within-trial temporal context, overall RT perseveration effects were noted (i.e., slower in both blocks when Block 1 contained S rather than M patterns). Such carryover effects suggest caution for interpreting patterns of RTs in blocked designs.

Fifth, do the present results generalize to visual sequences? Although attentional entrainment has been demonstrated with visual sequences (Martin et al., 2005), motor synchrony studies indicate greater variability with visual than with auditory sequences (Bartlett \& Bartlett, 1959; Chen, Repp, \& Patel, 2002; Patel et al., 2005; Repp \& Penel, 2002).

Exploration of these issues will add clarity to our understanding of how temporal structure influences temporal preparation and decision making.

\section{AUTHOR NOTE}

Thanks to Brad Havelka for help with data collection. This research was supported by a grant to R.J.E. from the Caroline B. Monahan Fund for Experimental Research Support in the Music Cognition/Perception Area within the Department of Psychology at The Ohio State University and by NSF Grant BCS-0738059 awarded to Trisha Van Zandt and M.R.J. Correspondence concerning this article should be addressed to R. J. Ellis, Beth Israel Deaconess Medical Center and Harvard Medical School, 330 Brookline Ave., Palmer 127, Boston, MA 02215 (e-mail: rellis@bidmc.harvard.edu).

\section{REFERENCES}

BARnes, R., \& Jones, M. R. (2000). Expectancy, attention, and time. Cognitive Psychology, 41, 254-311.

Bartlett, N. R., \& BartLetT, S. C. (1959). Synchronization of a motor response with an anticipated sensory event. Psychological Review, 66, 203-218

Bausenhart, K. M., Rolke, B., \& Ulrich, R. (2008). Temporal preparation improves temporal resolution: Evidence from constant foreperiods. Perception \& Psychophysics, 70, 1504-1514.

Bertelson, P. (1967). The time-course of preparation. Quarterly Journal of Experimental Psychology, 19, 272-279.

Bertelson, P., \& Tisseyre, F. (1968). The time-course of preparation with regular and irregular foreperiods. Quarterly Journal of Experimental Psychology, 20, 297-300.

Bregman, A. S. (1990). Auditory scene analysis: The perceptual organization of sound. Cambridge, MA: MIT Press.

Chen, Y., Repr, B. H., \& PAtel, A. D. (2002). Spectral decomposition of variability in synchronization and continuation tapping: Comparisons between auditory and visual pacing and feedback. Human Movement Science, 21, 515-532.

Correa, A., Lupiáñez, J., \& Tudela, P. (2005). Attentional preparation based on temporal expectancy modulates processing at the perceptual level. Psychonomic Bulletin \& Review, 12, 328-334.

Correa, A., Lupiáñez, J., \& Tudela, P. (2006). The attentional mechanism of temporal orienting: Determinants and attributes. Experimental Brain Research, 169, 58-68.

Correa, A., \& Nobre, A. C. (2008). Neural modulation by regularity and passage of time. Journal of Neurophysiology, 100, 1649-1655.

Correa, A., Sanabria, D., Spence, C., Tudela, P., \& Lupiáñez, J. 
(2006). Selective temporal attention enhances the temporal resolution of visual perception: Evidence from a temporal order judgment task. Brain Research, 1070, 202-205.

Coull, J. T., Frith, C. D., Büchel, C., \& Nobre, A. C. (2000). Orienting attention in time: Behavioral and neuroanatomical distinction between exogenous and endogenous shifts. Neuropsychologia, $\mathbf{3 8}$, 808-819.

Coull, J. T., \& Nobre, A. C. (1998). Where and when to pay attention: The neural systems for directing attention to spatial locations and to time intervals as revealed by both PET and fMRI. Journal of Neuroscience, 18, 7426-7435.

Doherty, J., Rao, A., Mesulam, M., \& Nobre, A. (2005). Synergistic effect of combined temporal and spatial expectations on visual attention. Journal of Neuroscience, 25, 8259-8266.

Drake, C., \& BotTe, M. C. (1993). Tempo sensitivity in auditory sequences: Evidence for a multiple-look model. Perception \& Psychophysics, 54, 277-286.

DrazIN, D. H. (1961). Effects of foreperiod, foreperiod variability and probability of stimulus occurrence on simple reaction time. Journal of Experimental Psychology, 62, 43-50.

ElLis, R. J., \& JoNES, M. R. (2009). The role of accent salience and joint accent structure in meter perception. Journal Experimental Psychology: Human Perception \& Performance, 35, 264-280.

Fraisse, P. (ED.) (1978). Time and rhythm perception. New York: Academic Press.

GARNER, W. R. (1974). The processing of information and structure. Potomac, MD: Erlbaum.

GRAHN, J. A., \& BRETT, M. (2007). Rhythm and beat perception in motor areas of the brain. Journal of Cognitive Neuroscience, 19, 893-906.

Grahn, J. A., \& BRETT, M. (2009). Impairment of beat-based rhythm discrimination in Parkinson's disease. Cortex, 45, 54-61.

Grondin, S. (2001). From physical time to the first and second moments of psychological time. Psychological Bulletin, 127, 22-44.

Grondin, S., \& RAMMSAYER, T. (2003). Variable foreperiods and temporal discrimination. Quarterly Journal of Experimental Psychology, 56A, 731-765.

HANDEL, S. (1992). The differentiation of rhythmic structure. Perception \& Psychophysics, 52, 497-507.

Jones, M. R. (1976). Time, our lost dimension: Toward a new theory of perception, attention, and memory. Psychological Review, 83, 323355 .

Jones, M. R. (2009). Musical time. In S. Hallam, I. Cross, \& M. Thaut, (Eds.), Oxford handbook of music psychology (pp. 81-92). Oxford: Oxford University Press.

Jones, M. R., \& Boltz, M. (1989). Dynamic attending and responses to time. Psychological Review, 96, 459-491.

Jones, M. R., Boltz, M., \& KidD, G. (1982). Controlled attending as a function of melodic and temporal context. Perception \& Psychophysics, 32, 211-218.

Jones, M. R., Johnston, H. M., \& Puente, J. (2006). Effects of auditory pattern structure on anticipatory and reactive attending. Cognitive Psychology, 53, 59-96.

Jones, M. R., Kidd, G., \& Wetzel, R. (1981). Evidence for rhythmic attention. Journal of Experimental Psychology: Human Perception \& Performance, 7, 1059-1073

Jones, M. R., \& YeE, W. (1997). Sensitivity to time change: The role of context and skill. Journal Experimental Psychology: Human Perception \& Performance, 23, 693-709.

KepPEL, G., \& WiCKens, T. D. (2004). Design and analysis: A researcher's handbook (4th ed.). Upper Saddle River, NJ: Prentice Hall.

KidD, G. R., Boltz, M., \& Jones, M. R. (1984). Some effects of rhythmic context on melody recognition. American Journal of Psychology, 97, 153-173.

KLeIN, R., \& KeRr, B. (1974). Visual signal detection and the locus of foreperiod effects. Memory \& Cognition, 2, 431-435.

Klemmer, E. T. (1956). Time uncertainty in simple reaction time. Journal of Experimental Psychology, 51, 179-184.

Lange, K., \& HeIL, M. (2008). Temporal attention in the processing of short melodies: Evidence from event-related potentials. Musicea Scientiae, 12, 27-48.

LARGe, E. W. (2010). Neurodynamics of music. In M. R. Jones, A. N. Popper, \& R. R. Fay (Eds.), Music perception (pp. 201-231). New York: Springer.
Large, E. W., \& Jones, M. R. (1999). The dynamics of attending: How people track time-varying events. Psychological Review, 106, 119159

Large, E. W., \& Kolen, J. F. (1994). Resonance and the perception of musical meter. Connection Science, 6, 177-208.

LERDAHL, F., \& JACKENDOFF, R. (1983). A generative theory of tonal music. Cambridge, MA: MIT Press.

London, J. M. (2004). Hearing in time: Psychological aspects of musical meter. New York: Oxford University Press.

Los, S. A., \& SchUT, M. L. (2008). The effective time course of preparation. Cognitive Psychology, 57, 20-55.

LuCE, R. D. (1986). Response times: Their role in inferring elementary mental organization. New York: Oxford University Press.

Martin, T., Egly, R., Houch, J. M., Bish, J. P., Barrera, B. D., Lee, D. C., \& Tesche, C. D. (2005). Chronometric evidence for entrained attention. Perception \& Psychophysics, 67, 168-184.

Martin, T., Houck, J. M., Bish, J. P., Kičıč, D., Woodruff, C. C., Moses, S. N., ET AL. (2006). MEG reveals different contributions of somatomotor cortex and cerebellum to simple reaction time after temporally structured cues. Human Brain Mapping, 27, 552-561.

McAuley, J. D. (2010). Tempo and rhythm. In M. R. Jones, A. N. Popper, \& R. R. Fay (Eds.), Music perception (pp. 165-199). New York: Springer.

McAuley, J. D., \& Jones, M. R. (2003). Modeling effects of rhythmic context on perceived duration: A comparison of interval and entrainment approaches to short-interval timing. Journal of Experimental Psychology: Human Perception \& Performance, 29, 1102-1125.

McAuley, J. D., \& KidD, G. R. (1998). Effect of deviations from temporal expectations on tempo discrimination of isochronous tone sequences. Journal of Experimental Psychology: Human Perception \& Performance, 24, 1786-1800.

McAuley, J. D., \& Miller, N. S. (2007). Picking up the pace: Effects of global temporal context on sensitivity to the tempo of auditory sequences. Perception \& Psychophysics, 69, 709-718.

McAuley, J. D., \& Semple, P. (1999). The effect of tempo and musical experience on perceived beat. Australian Journal of Psychology, 51, 176-187.

Müller-Gethmann, H., Ulrich, R., \& Rinkenauer, G. (2003). Locus of the effect of temporal preparation: Evidence from the lateralized readiness potential. Psychophysiology, 40, 597-611.

NIEMI, P., \& NÄÄTÄNEN, R. (1981). Foreperiod and simple reaction time. Psychological Bulletin, 89, 133-162.

OLSON, I., \& CHUN, M. (2001). Temporal contextual cuing of visual attention. Journal of Experimental Psychology: Learning, Memory, \& Cognition, 27, 1299-1313.

Patel, A. D., \& Daniele, J. R. (2003). An empirical comparison of rhythm in language and music. Cognition, 87, B35-B45.

Patel, A. D., Iversen, J. R., Chen, Y., \& Repp, B. H. (2005). The influence of metricality and modality on synchronization with a beat. Experimental Brain Research, 163, 226-238.

Patel, A. D., Iversen, J. R., \& Rosenberg, J. C. (2006). Comparing the rhythm and melody of speech and music: The case of British English and French. Journal of Acoustical Society of America, 119, 3034-3047.

PfordResher, P. Q. (2003). The role of melodic and rhythmic accents in musical structure. Music Perception, 20, 431-464.

Povel, D.-J., \& Essens, P. (1985). Perception of temporal patterns. Music Perception, 2, 411-440.

Povel, D.-J., \& OKKerman, H. (1981). Accents in equitone sequences. Perception \& Psychophysics, 30, 565-572.

Praamstra, P., Kourtis, D., KwoK, H. F., \& Oostenveld, R. (2006). Neurophysiology of implicit timing in serial choice reaction-time performance. Journal of Neuroscience, 26, 5448-5455.

RATCLIFF, R. (1993). Methods for dealing with reaction time outliers. Psychological Bulletin, 114, 510-532.

RepP, B. H., \& Penel, A. (2002). Auditory dominance in temporal processing: new evidence from synchronization with simultaneous visual and auditory sequences. Journal of Experimental Psychology: Human Perception \& Performance, 28, 1085-1099.

Requin, J., Grandjon, M., Durup, H., \& Reynard, G. (1973). Effects of a timing signal on simple reaction time with a rectangular distribution of foreperiods. Quarterly Journal of Experimental Psychology, 25, 344-353. 
Schmidt-Kassow, M., Schubotz, R. I., \& Kotz, S. A. (2009). Attention and entrainment: P3b varies as a function of temporal predictability. NeuroReport, 20, 31-36.

Simon, J. R., \& Slaviero, D. P. (1975). Differential effects of a foreperiod countdown procedure on simple and choice reaction time. Journal of Motor Behavior, 7, 9-14.

Snyder, J. S., \& Krumhansl, C. L. (2001). Tapping to ragtime: Cues to pulse finding. Music Perception, 18, 455-489.

Steinborn, M. B., Rolke, B., Bratzke, D., \& Ulrich, R. (2008). Sequential effects within a short foreperiod context: Evidence for the conditioning account of temporal preparation. Acta Psychologica, 129, 297-307.

Temperley, D. (2001). The cognition of basic musical structures. Cambridge, MA: MIT Press.

TodD, R., Boltz, M. G., \& Jones, M. R. (1989). The MIDILAB auditory research system. Psychomusicology, 8, 17-28.

Woodrow, H. (1914). The effect upon reaction time of variation in the preparatory interval. In The measurement of attention [Monograph] (pp. 16-65). Princeton, NJ: Psychological Review.

\section{NOTES}

1. Although the FP interval is traditionally defined as an interstimulus interval (ISI), we manipulate it in this research as an interonset interval (IOI). However, in practice, if warning stimuli are brief and of constant duration, differences between ISI and IOI are minimal (see Los \& Schut, 2008, for a discussion).

2 . The best clock grid is defined as the one with the least counterevidence, $C$, defined by Povel and Essens (1985) as $C=(4 s+u)$, where $s$ refers to a clock tick falling on a silence and $u$ a clock tick falling on an unaccented tone. Accents were defined according to Povel and Okkerman (1981): isolated tones, the second tone of a two-tone group, and the first and last tones of longer groups. Assuming a clock with a unit of $500 \mathrm{msec}$ that begins on the first tone, the $\mathrm{S}$ patterns in Figure 1B have $C$ s ranging from 16 to 25, and all of the M patterns in Figure 1A have $C$ s of 12. The Spearman correlation between FP and $C$ is significant neither for the M patterns $(\rho=0)$ nor for the $\mathrm{S}$ patterns $(\rho=-.242, p=.643)$.

3. Although we discuss the U-shaped profile in terms of longer RTs at the 1,000-msec FP, an alternative explanation is a shortening of RTs at the 500-msec FP for both the $\mathrm{M}$ group and the $\mathrm{S}$ group. That is, in Figure 4B, the mean RT at the 500-msec FP was shorter than predicted by a linear relationship defined by RTs at 250 -msec and $1,000-\mathrm{msec}$ FPs by $44 \mathrm{msec}$ in the $\mathrm{M}$ group and $52 \mathrm{msec}$ in the $\mathrm{S}$ group. Although we restrict discussion of this interpretation to this note, at the middle FP $(500 \mathrm{msec})$, the mean RT in the S group $(660 \mathrm{msec})$ was nearly identical to the statistical mean of IOIs in patterns $(667 \mathrm{msec})$.

4. This DAT interpretation does not preclude another factor, which depends on metrical relations. The metric binding hypothesis (Jones, 2009) implies that $M$ rhythms promote quicker perceptual learning than $\mathrm{S}$ rhythms. Thus, pattern-specific learning of individual rhythms will also contribute to a listener's anticipations of final FPs in M patterns. The role of time hierarchies in perceptual learning of rhythms deserves investigation.

5. This hypothesis could be further tested. For example, if the last two or three IOIs in a pattern (including the FP) all had the same duration, this should lead to increased activation of the congruent oscillator (relative to a single instance of that oscillator's period). In such a design, we would expect exaggerated differences in RT as a function of FP (shorter for $250 \mathrm{msec}$ and longer for 1,000). Unfortunately, the present set of stimuli (Figures 1A an 1B) preclude a systematic assessment of this hypothesis here; only the 500-msec IOI appears as the last (pattern M2) or the last two (pattern M5) IOIs. We thank John Iversen for this suggestion.

(Manuscript received September 7, 2009; revision accepted for publication June 9, 2010.) 